INPLASY

PROTOCOL

To cite: Zhao et al. Efficacy of Biqi Capsule in the Adjuvant Treatment of Ankylosing Spondylitis : A Systematic Review and Meta-Analysis. Inplasy protocol 202230009. doi:

10.37766/inplasy2022.3.0009

Received: 02 March 2022

Published: 02 March 2022

Corresponding author:

Pan-Pan Zhao

lyhzyk@163.com

Author Affiliation:

Affiliated Hospital of Nankai University (Tianjin Fourth Hospital).

Support: No financial support.

Review Stage at time of this submission: Formal screening of search results against eligibility criteria.

\section{Efficacy of Biqi Capsule in the Adjuvant Treatment of Ankylosing Spondylitis : A Systematic Review and Meta-Analysis}

Zhao, PP1; Li, YH²; Xia, DQ³ .

Review question / Objective: To evaluate the efficacy of Biqi capsule in the adjuvant treatment of patients with AS. And provide the latest evidence-based medical evidence for the clinical application of Biqi capsule in the treatment of AS.

Condition being studied: Ankylosing spondylitis (AS) is a chronic autoimmune disease that mainly involves the spinal column, sacroiliac and other axial joints, and also involves multiple extrarticular systems. Currently, there is no specific treatment plan, and the disability rate is very high. At present, some clinical studies have shown that Biqi capsule is safe and effective in the adjuvant treatment of ankylosing spondylitis, but there is a lack of systematic review. This study aims to systematically evaluate the efficacy of Biqi capsule in the adjuvant treatment of ankylosing spondylitis, and to provide evidence for the clinical application of Biqi capsule in the treatment of AS.

INPLASY registration number: This protocol was registered with the International Platform of Registered Systematic Review and Meta-Analysis Protocols (INPLASY) on 02 March 2022 and was last updated on 02 March 2022 (registration number INPLASY202230009).

Conflicts of interest:

None declared.

\section{INTRODUCTION}

Review question / Objective: To evaluate the efficacy of Biqi capsule in the adjuvant treatment of patients with AS. And provide the latest evidence-based medical evidence for the clinical application of Biqi capsule in the treatment of AS.

Condition being studied: Ankylosing spondylitis (AS) is a chronic autoimmune disease that mainly involves the spinal column, sacroiliac and other axial joints, 
and also involves multiple extrarticular systems. Currently, there is no specific treatment plan, and the disability rate is very high. At present, some clinical studies have shown that Biqi capsule is safe and effective in the adjuvant treatment of ankylosing spondylitis, but there is a lack of systematic review. This study aims to systematically evaluate the efficacy of Biqi capsule in the adjuvant treatment of ankylosing spondylitis, and to provide evidence for the clinical application of Biqi capsule in the treatment of AS.

\section{METHODS}

Participant or population: The participants should be diagnosed with AS by using clearly defined or internationally recognized criteria.

Intervention: The treatment of the experimental group was treated with Biqi capsule on the basis of conventional drug treatment.

Comparator: The control group received conventional medication.

Study designs to be included: We will include RCTs of Biqi capsule combined with western medicine in the treatment of AS, which should evaluate at least one primary outcome.

Eligibility criteria: Patients with AS, age and sex were not restricted. Biqi capsule combined with conventional drug treatment were used as intervention measures in the experimental group, and conventional drug treatment were used as control measures.

Information sources: We will use 8 databases to search all relevant literature resources. Including three English databases PubMed, Embase and Cochrane Library, and four Chinese databases China National Knowledge Infrastructure database (CNKI), Wanfang Chinese digital periodical and conference database (WanFang Data), VIP Chinese Science and Technique Journals Database (VIP) and
China Biomedical Literature Service System (SinoMed).

Main outcome(s): The outcome measures we focused on included efficacy indicators (ASAS20, ASAS40), Bath ankylosing spondylitis disease activity index (BASDAI), Bath Ankylosing Spondylitis Functional Index (BASFI), Bath Ankylosing Spondylitis Metrology Index (BASMI), Visual Analogue Scale(VAS), erythrocyte sedimentation rate (ESR), C protein response (CRP), and adverse reactions.

Quality assessment / Risk of bias analysis: To assess the bias of the included literature, we will use the Cochrane risk assessment tool. Its main content includes the following 7 items: random method selection; allocation hiding; blind method, completeness of the result data; whether the evaluator is blind; selectively reporting results; other biases. The above 7 items all contain 3 options of "yes", "no" and "unclear".

Strategy of data synthesis: RevMan V.5.4.1 software will be used for meta-analysis. Dichotomous variables will be expressed using the risk ratio (RR) with $95 \%$ confidence interval $(\mathrm{Cl})$ and continuous variables by the mean difference (MD) or standardized mean difference (SMD) with $95 \% \mathrm{Cl}$. The Q-test and 12 values will be applied to measure the inter-study heterogeneity. When the $\mathrm{p}$-value of Q-test $>0.1$ and $12<50 \%$, heterogeneity is acceptable and a fixed effects model will be applied. If $p$-value of $Q$-test $<0.1$ and 12 $>50 \%$, heterogeneity is significant and subgroup analysis will be developed to investigate the possible sources according to the characteristics of study, types of intervention and controls, different outcome measurements and study quality, if still unable to find, we will use random effect model to estimate or descriptive analysis.

Subgroup analysis: If necessary, subgroup analyses will be performed to investigate possible sources of heterogeneity according to the characteristics of study, 
types of intervention and controls, different outcome measurements and study quality.

Sensitivity analysis: To assess the influence of each individual study, leave-one-out sensitivity analysis was performed iteratively by removing one study at a time to confirm that the findings were not influenced by any single study.

Country(ies) involved: China.

Keywords: Biqi capsule; ankylosing spondylitis; systematic review; metaanalysis.

Contributions of each author:

Author 1 - Pan-Pan Zhao.

Author 2 - Yan-Hua Li.

Author 3 - Dong-Qin Xia. 